\title{
Risk management overview of tunnels using numerical modeling
}

\begin{abstract}
Purpose: The purpose of this paper is to introduce the numerical methods in tunnel engineering and their capabilities to indicate the fracture and failure in all kinds of tunneling methods such as New Austrian Tunneling Method, tunnel boring machine and cut-cover. An essential definition of numerical modeling of tunnels to determine the interaction between geo-material (soil and rock) surrounding the tunnel structure is discussed.

Design/methodology/approach: Tunnel geo-material (soil and rock) interaction requires advanced constitutive models for the numerical simulation of linear, nonlinear, timedependent, anisotropic, isotropic, homogenous and nonhomogeneous behaviors. The numerical models discussed in this paper are developed in finite element method (FEM), finite deference method (FDM), boundary element method and discrete element method and these tools are used to illustrate the behavior of tunnel structure deformation under different loads and in complicated conditions. The disadvantage of this method is the tunnel lining assumed an independent structure under fixed load which is unable to model soil-lining interaction. Predicting the effect of all natural factors on tunnels is the most difficult method. The above-mentioned numerical methods are very simple and quick to use and the results are conservative and practical for users. One of the most significant advantages of the numerical method is in predicting the critical area surrounding the tunnel and the tunnel structure before making the tunnel construction due to different loads.

Findings: Numerical modeling is used as control method in reducing the risk of tunnel construction failures. Since some factors such as settlement and deformation are not completely predictable in rock and soil surrounding the tunnel, using numerical modeling is a very economical and capable method in predicting the behavior of tunnel structures in various complicated conditions of loading. Another benefit of using numerical simulation is in the colorful illustrations predicting the tunnel behavior before, during and after construction and operation.

Originality/value: There are not many conducted studies using numerical models to tunnel structures that estimate the critical zones. As some of the methods available have limitation in simulating and modeling the whole tunnel design factors, numerical modeling seems to be the best option, because it is fast, economical, accurate and more interesting in predicating critical zones in tunnel. However, what softwares predict are not always the same as real ground nature conditions in which there is tunnel.
\end{abstract}

Keyword: Civil engineering; Modelling; Numerical analysis; Risk management; Tunnels 